\title{
Teaching with/out the F-Word
}

\section{Ein autoethnografischer Trialog auf der Suche nach einer feministischen Lehrhaltung}

\author{
Sabine Klinger, Daniela Jauk \& Nicole Pruckermayr
}

Journal für Psychologie, 27(1), 30-50

https://doi.org/10.30820/0942-2285-2019-1-30

www.journal-fuer-psychologie.de

\section{Zusammenfassung}

In diesem Beitrag denkt und schreibt das interdisziplinäre Autor*innenteam im Rahmen von autoethnografischen Trialogen zum Thema feministische Lehre beziehungsweise Lehren inner- und außerhalb der Geschlechterforschung. Verbindend ist dabei die Suche nach einer feministischen Lehrhaltung und -praxis, die mit und/oder ohne den Begriff Feminismus respektive Feminismen gelingen kann. Diese Diskussion erfolgt vor dem Hintergrund verschiedener disziplinärer Perspektiven der Autor*innen. Angeregt wurde dieser Austausch durch voneinander unabhängige Erfahrungen der Autor*innen, dass das Wort Feminismus (FWort) bei unterschiedlichen Akteur*innengruppen (z. B. Studierende, Fördergeber*innen) im Kontext von Lehre und Forschung auf Widerstand und Unbehagen stößt. Diese Erfahrungen werden im Beitrag mittels theoretischer Referenzpunkte kontextualisiert. Dabei fokussieren wir auf aktuelle Gesellschaftsdiagnosen, die aktuelle Genderdiskurse als »rhetorische Modernisierung « (Wetterer 2003) identifizieren und einen »neuen Geschlechtervertrag « (McRobbie 2010) konstatieren, welche antifeministische Tendenzen beinhalten und wo Feminismen als etwas Überholtes in die Vergangenheit verlegt werden (McRobbie 2010). Vor dem Hintergrund dieser gesellschaftlichen Rahmenbedingungen diskutieren wir in diesem Beitrag die Frage, ob feministische Lehre und Forschung möglich oder notwendig ist, ohne das F-Wort anzusprechen und somit subversiv zu agieren. Wir wollen in diesem Beitrag bewusst nicht feministische Wissenschaft als Kanon oder Teil eines Kanons präsentieren, sondern zeigen in unseren Trialogen, dass wir Wissenschaft als historisch entlang von patriarchalen Machtlinien gewachsen verstehen, und es wichtig und notwendig ist, in die Gestaltung der Wissenschaft als soziale Produktion einzugreifen. Mit und ohne F-Wort.

Schlüsselwörter: Autoethnografie, feministische Pädagogik, Lehre, Interdisziplinarität, Geschlechterforschung 


\section{Summary}

\section{Teaching with/out the F-Word}

In this paper the interdisciplinary team of authors analyzes a series of autoethnographic trialogues addressing feminist pedagogy, respectively teaching within and beyond gender studies environments. The authors find common ground in their search for a feminist stance in teaching and educational practices which may work with or without making the concept of feminism(s) explicit. The conversations evolved against the backdrop of the diverse disciplinary backgrounds of the authors and the universal experience by the authors that the mere mention of the word feminism (s) (the F-Word) evokes resistance among different actors (students, funders, etc.) in the field of education. We contextualize our experiences within the framework of $\gg$ rhetorical modernization « (Wetterer 2003) and the »new gender contract « (McRobbie 2010) which both denote a re-traditionalization of gender discourses. Keeping in mind these contemporary developments we discuss if feminist teaching and research is necessary, and if it might be a legitimate strategy to avoid the F-Word and act more subversively. It is not our goal in this paper to present feminist research and teaching as a canon or part of a canon; instead we have developed an awareness in our trialogues that science is historically constructed along axes of inequality. Because of this fact it is particularly important to intervene in this ongoing social production. With and without the F-Word.

Keywords: autoethnography, feminist pedagogy, teaching, interdisciplinarity, gender studies

\section{Einleitung}

Wissenschaftliches Arbeiten ist ein zutiefst vergeschlechtlichter Prozess und wird als solcher auch in der Lehre rekonstruiert und multipliziert. Die Reflexion und Offenlegung eines heteronormativen und patriarchalen Bias in Wissensproduktionsprozessen sind essenzielle Strategien feministischer Wissenschaftskritik und feministischer Lehre. Aber was macht feministische Lehre aus, die im Spannungsfeld von wissenschaftlichem Arbeiten und gesellschaftskritischem Impetus angesiedelt ist? Wie lassen sich Feminismen und feministische Inhalte in die Lehre integrieren und thematisieren? Wie gestaltet sich dabei das Verhältnis von Didaktik und feministischer Wissenschaft? Das sind einige der Fragen, denen das interdisziplinäre Autor*innenteam in diesem Beitrag nachgeht. Die im folgenden dargestellte Diskussion fußt auf einer Serie von dialogischen (eigentlich trialogischen) kritischen Reflexionen, Vorträgen und Gesprächen, die von 2016 bis 2018 stattgefunden haben und nach wie vor andauern. In diesen strukturierten Dialogen haben wir unsere feministische Lehrpraxis autoethnografisch hinterfragt und über disziplinäre, aber auch interdisziplinäre Zugänge, Positionen und Erfahrungen aus unserer Lehrpraxis debattiert. Unsere Diskussionen, aber auch die folgende Darstellung 
dieser sind bestimmt von der feministischen Praxis des Situierten Wissens von Donna Haraway (1995). Dabei geht es uns darum, die Relevanz der jeweiligen subjektiven Perspektive anzuerkennen und durch Verknüpfungen verschiedener Perspektiven eine angenäherte Objektivität zu erreichen. Es geht letztlich um ein Eingeständnis, dass es Objektivität nicht gibt und eine vermeintliche Objektivität von Wissenschaft als Machtinstrument eingesetzt wird. Haraway $(1995,579)$ macht klar:

»Feminists don't need a doctrine of objectivity that promises transcendence, a story that loses track of its mediations just where someone might be held responsible for something, and unlimited instrumental power. We don't want a theory of innocent powers to represent the world, where language and bodies both fall into the bliss of organic symbiosis.«

Ganz in diesem Sinne versuchen wir hier nicht eine Theorie feministischer Lehre zu destillieren oder eine feministische Leistungsschau zu inszenieren. Wir diskutierten in unseren Trialogen vielmehr die feministische Praxis unserer jeweiligen eigenen sozialen Positionen, um Privilegien und die eigenen Schwachstellen zu bearbeiten und uns bewusst zu sein, dass unseres ein lokales, ein begrenztes Wissen ist, welches eben nicht für alle sprechen kann, welches aber eine gemeinsame Annäherung schafft. Denn »feminist objectivity means quite simply situated knowledges « (Haraway 1995, 581). Die verschiedenen Perspektiven sind auch relevant, um darauf hinweisen zu können, dass die unterschiedlichen Disziplinen keine strukturell anderen Herangehensweisen an feministische Lehre evozieren und dass wir uns solidarisieren können und sollen über Disziplinengrenzen hinweg. Zudem thematisieren wir hier, wie Formen von (Un-)Gleichheit, Differenz, Herrschaft, Ein- und Ausgrenzung unter feministischer Perspektive in der Lehre des wissenschaftlichen Arbeitens positioniert werden können. Letzteres beschäftigt uns besonders, da wir in Lehrpraxis und -erfahrungen immer wieder (nicht nur vonseiten der Studierenden) auf rhetorische Modernisierungsprozesse (Wetterer 2003, 2013) und eine »Politik der Desartikulation « (McRobbie, 2010) stoßen beziehungsweise diese sichtbar werden und wir uns die Frage stellen, wie wir produktiv damit umgehen können, ohne Abwehr und Widerstand zu provozieren. Laut Angela McRobbie wird heute jungen Frauen ein neuer Geschlechtervertrag angeboten, der die Desartikulation feministischer Forderungen und Inhalte im $\gg$ neuen Gender-Diskurs « (2010) beinhaltet und im Gegenzug Frauen das Angebot macht, öffentlich sichtbar zu werden, um am Arbeits- und Bildungsmarkt zu partizipieren und an der Konsumkultur und Bürgergesellschaft teilzunehmen (2010). Vor diesem Hintergrund beschäftigen wir uns zudem mit der Frage, ob und wie es beim Einbringen feministischer Inhalte in die Lehre wissenschaftlichen Arbeitens oder auch in die künstlerische Produktion Sinn machen kann, das F-Wort (Feminismus) gerade nicht offensiv zu verwenden und dennoch subversiv feministisch zu bilden und zu inspirieren. Damit 
meinen wir eine feministische Lehrhaltung, die das Sprichwort action speak louder than words ernst nimmt. Das heißt, eine Lehrhaltung zu verfolgen, die in Methoden und Didaktik feministisch geprägt ist, nicht nur in Texten und Lehrmaterialien.

Die Leser*innen, die sich im Folgenden einen linearen und klassischen Aufsatz erwarten, der interpretativ-hermeneutischen akademischen Formeln folgt, werden von unserem Beitrag überrascht sein, da dieser - wie auch die feministische Lehre selbst von Multivokalität, Unstimmigkeiten und Zwischenräumen geprägt ist, (Selbst-)Kritik anregen soll und im Sinne des Situierten Wissens (Haraway 1995) Anknüpfungspunkte für feministische (Lehr-)Praxen bieten soll. Wir gehen davon aus, in Lehr-/Lernräumen auf aktive und eigenständige Personen zu treffen. In Teaching to Transgress (hooks 1994) lernen wir, dass Lehre und Lernen auch heißt, verschiedenen Wissensarten Raum zu geben und auch Verbindungen zwischen akademischem Wissen und Lebensrealitäten zuzulassen und einzubringen. Der optimale Lernraum ist nicht existent, aber wir wollen uns ihm annähern. Mittels der Autoethnografie als Forschungsmethode und als Form wissenschaftlichen Schreibens wagen wir den Versuch der systematischen Introspektion und einer dreistimmigen Selbstreflexion/-befragung (Ellis und Bochner 2000; Ellis et al. 2011; Ploder und Stadlbauer 2013), um in einen Dialog mit den Rezipient*innen zu treten. In unserer Auseinandersetzung geht es also nicht darum, ein Konzept, ähnlich einer Vorlage, für die perfekte und universale feministische Lehre zu erarbeiten, sondern Strategien zu diskutieren und Themen zu beleuchten, die Anregungen für eine kritisch-feministische Lehrhaltung geben. Wie bereits bell hooks (2010) geht es uns um eine Lehrhaltung, die ermächtigt, die es Lernenden und Lehrenden ermöglicht, sich kritisch mit der sie umgebenden Welt auseinanderzusetzen und diese im nächsten Schritt zu verändern. Nicht nur innerhalb unserer Lehre sind wir überzeugt davon, mit konstruktiven Kritiken und einem »Im-Gespräch-Bleiben « herausfordernde (gesellschaftliche) Konfliktsituationen lösen zu können, die nicht immer, aber oft auf fehlender Geschlechtergerechtigkeit oder Dethematisierung von unterschiedlichen Bedürfnissen unterschiedlicher Geschlechter fußen.

\section{Wege und Zugänge der Autor*innen}

Auch wenn es sich bei diesem Text um eine theoretisch durchzogene Autoethnografie handelt, werden im Beschreiben der eigenen Erfahrungen, des subjektiven Erlebens die Grenzen zwischen Therapeutischem, Politischem und Wissenschaftlichem überschritten (Ellis und Bochner, 2000). Dabei geht es weniger um das Ausbreiten unserer eigenen Gefühle als Lehrende, Forschende und Kunstschaffende, sondern darum, die eigenen verkörperten Erfahrungen als Quelle von Wissen und Veränderung für die feministische Lehre zu nehmen. bell hooks illustriert dies, auf Basis ihrer eigenen Er- 
fahrungen, folgendermaßen: »Those classrooms were the one space where teachers were willing to acknowledge a connection between ideas learned in university settings and those learned in life practices « $(1994,15)$. Die Auseinandersetzung mit (eigenen und fremden) Gefühlen und Erfahrungen ist dabei unvermeidlicher und unverzichtbarer Bestandteil des Forschungs- und Schreibprozesses (Ploder and Stadlbauer 2013, 375).

Für die interdisziplinäre Auseinandersetzung mit und (Selbst-)Reflexion über feministische(n) Konzeptionen von Lehre und von (künstlerischen) Interventionen in der Praxis wissenschaftlichen Arbeitens ist die Autoethnografie besonders geeignet, da sie an den Grenzen zwischen Wissenschaft und Kunst operiert und damit auch an den Grenzen des jeweiligen Faches beziehungsweise der jeweiligen Disziplin (Ploder and Stadlbauer 2013, 401). Die Auseinandersetzung mit und ein Denken an diesen Grenzen kann zu neuen Erkenntnissen inspirieren und das (Selbst-)Verständnis als Lehrende, Forschende und Kunstschaffende schärfen und akzentuieren. Diese Grenzen, egal ob von Disziplinen oder vom eigenen Selbstverständnis, können nicht nur überschritten, sondern auch verschoben werden. Zudem sehen wir bereits in unserer Wahl der Autoethnografie als Forschungsmethode und als Form wissenschaftlichen Schreibens einen wesentlichen (subversiven) feministischen Impetus, da hiermit die Reflexion von Macht und Hierarchie mit einem emanzipatorischen Anspruch belegt und verknüpft wird (Ploder und Stadlbauer 2013, 395ff.). Unsere Trialoge sind dabei gleichzeitig Prozess und Produkt der Forschung (Ellis et al. 2011).

Wir, die Autor*innen, unterrichten in verschiedenen Konstellationen, an verschiedenen deutsch- und englischsprachigen Universitäten, in Gender Studies und auch in anderen Disziplinen wie Soziologie, Erziehungs- und Bildungswissenschaft, Kunst/Architektur, die wir ins unseren Trialogen normative Klassenzimmer nennen. In diesen Lehr- und Lernräumen steht die Reflexion von gesellschaftlichen Normen, Ordnungen und Hierarchien nicht im Zentrum, andere Wissensfragen werden fokussiert. Durch Vernachlässigen der Frage nach den Implikationen von Geschlechtlichkeit oder (im schlimmsten Fall) durch nichtreflektierendes, sondern normierendes Handeln gegenüber den Studierenden (z.B. durch Beharren auf Geschlechterdichotomie) werden unreflektierte Normative wiederholt und vertieft. Normativ kann auch in Bezug auf die Dichotomie zwischen den Lehrenden, die immer in hierarchischer Beziehung zu den Studierenden gesetzt sind, bedeuten. Das Aussprechen und die Reflexion darüber, in welchem Setting sich der Klassenraum befindet, wirkt hier klärend. Im nichtnormativen Klassenzimmer erkennen wir die Wichtigkeit von (Selbst-)Reflexivität als wesentliches Merkmal der feministischen Forschung (Hesse-Biber und Piatelli 2012) und Lehre (Schlüter und Justen 2009) an.

Wir haben uns zur Vorbereitung der 4. Jahrestagung der Österreichischen Gesellschaft für Geschlechterforschung versammelt und begonnen, uns Fragen zu unserer Lehrpraxis 
zu stellen. Wir begannen Gespräche über die Fragen zu führen: Was zeichnet eine feministische Lernumgebung aus? Ist der Unterricht notwendigerweise feministisch, wenn wir über Geschlecht im Unterricht diskutieren? Wie können wir Feminismen und feministische Ideen im Unterricht über die Geschlechterforschung hinaus transportieren? Fühlen wir uns wohl mit dem in jedem Unterrichtskontext? Gibt es Situationen, in denen es strategisch sinnvoller ist, das F-Wort (Feminismen) (nicht) zu nutzen? Wir trafen uns zu internen Werkstattgesprächen und nahmen diesen gemeinsamen, praxisbezogenen Erfahrungsaustausch mit einem digitalen Aufnahmegerät auf. Unsere Trialoge und Diskussionen waren strukturiert durch die obigen Fragen und wurden transkribiert und dadurch zu Daten, die neue Fragen und Themen aufgeworfen haben. Basierend auf diesen Forschungsgesprächen haben wir auf der 4. Jahrestagung der Österreichischen Gesellschaft für Geschlechterforschung (2016) eine erste Analyse vorgestellt. Der vorliegende Artikel enthält das kritische Feedback, das wir beim Publikum auf der Konferenz erhalten haben, sowie eine Analyse weiterer Dialoge, die wir als Team und als wissenschaftliche Aktivist*innen in einer lokalen Radiosendung (Radio Helsinki 2017) geführt haben. Um unsere folgenden Ausführungen über das und Fragen nach dem Einbringen feministischer und gesellschaftskritischer Inhalte in die Lehre, in das wissenschaftliche Arbeiten und in Kunstproduktionen besser nachvollziehen zu können, braucht es aus unserer Sicht auch eine Vorstellung des Autor ${ }^{*}$ innenteams und ihrer jeweiligen individuellen Hintergründe in unseren je eigenen Stimmen:

Autor ${ }^{*}$ in L:Ich bin eine verpartnerte, kinderlose Cis-Frau und promovierte Erziehungsund Bildungswissenschaftlerin, die aus einer Arbeiter ${ }^{*}$ innenfamilie stammend als erstes Mitglied der Familie ein Hochschulstudium abgeschlossen hat und nun auch an der Universität lehrend und forschend tätig ist. Meine thematischen Schwerpunkte sind feministische Geschlechter- und Migrationsforschung und Prozesse der Digitalisierung in der Sozialen Arbeit. Mein Interesse an der Kategorie Geschlecht und den Zugang zu diesem Thema möchte ich hier kurz skizzieren: Ich selbst habe Erziehungs- und Bildungswissenschaften sowie Gender Studies studiert und mich bereits seit den Anfängen meines Studiums mit Geschlechterfragen beschäftigt. Durch den Besuch zahlreicher Seminare, Diskussionen mit Kommiliton ${ }^{*}$ innen, Familie und Freund*innen sowie die Wahl, in geschlechterbezogenen Arbeitsfeldern pädagogisch tätig zu sein, fand eine Vertiefung in die Thematik statt. Außerdem war und ist es für mich selbstverständlich, dass ich mich mit Geschlechterfragen, -verhältnissen und -differenzen beschäftige und normative, hierarchische, polare Vorstellungen von Geschlechterrollen und -ordnungen kritisch hinterfrage. Im Austausch mit anderen habe ich aber bemerkt, dass diese Betrachtung von Geschlecht als (pädagogisch) relevante Kategorie längst nicht von allen geteilt wird und meine Position oftmals der Legitimation bedarf und als indivi- 
duell geprägtes Interesse beziehungsweise individuelle Orientierung gesehen wird. Für mich kristallisierte sich zunehmend die Frage heraus, warum die feministische Thematisierung von Geschlecht im erziehungswissenschaftlichen Denken und pädagogischen Handeln oftmals als obsolet betrachtet wird, wenn (aus meiner Perspektive) ein reflektierender Umgang mit Geschlechtern (noch) nicht Teil (sozial-)pädagogischer Praxis ist.

Autor*in X: Ich war circa 13 Jahre alt, als ich mit rotem Autolack ein Frauenzeichen auf meine grüne 70er-Jahre-Schilftapete meines Kinderzimmers gemalt habe, zum Entsetzen meiner Eltern. Das Zimmer befand sich in einem Einfamilienhaus in einem steirischen Dorf, in dem es kein Geschäft, dafür aber viel familiäre Gewalt, Alkoholismus, und - nebst vielen Bauernhöfen - scheinbar nur einen Arbeiter*innenhaushalt gab: unseren. Ich habe mich mit dieser Wandmalerei der feministischen Arbeit verschrieben. In meiner Arbeit als Sozialarbeiterin und in meiner politischen Arbeit als Frauenbeauftragte der Stadt Graz habe ich viele individuelle Lebensgeschichten von Frauen, Mädchen und Transgender-Personen gesammelt, hinsichtlich derer ich später versucht habe, sie in einem soziologischen Rahmenwerk zu verstehen. Ich habe meinen $\mathrm{PhD}$ in Soziologie in den USA absolviert, wo ich nun verheiratet und Mutter eines Kindes bin - eine heteronormative Patina für eine queerfeministische Identifikation und Kalibrierung. Ich habe in den letzten Jahren an verschiedensten Universitäten in Österreich und den USA unterrichtet, komme aber aus der feministischen Gruppenarbeit mit Mädchen und habe Erfahrung in Erwachsenenbildung und Workshops. Derzeit versuche ich Biogärten in Frauengefängnissen im Auftrag eines »feministischen participatory action research frameworks « zu bauen. Hierbei bewege ich mich in dem Spannungsfeld einerseits Teil eines normierenden und disziplinierenden »prison industrial complex « zu sein und andererseits auch gegen dieses System zu arbeiten, es zu unterlaufen oder zumindest zu erodieren.

Autor*in O: Ich arbeite derzeit freiberuflich als Künstlerin, Kulturarbeiterin und in der Forstwirtschaft, habe Biologie und Architektur studiert, bin promoviert im Bereich Visuelle Kultur und Kulturanthropologie. Ich bin verheiratet und habe ein Kind, habe an verschiedenen österreichischen Universitäten unterrichtet, meist in der Regellehre im Fachbereich Zeitgenössische Kunst, aber oft anknüpfend an gendersensible Thematiken. Im Nachhinein würde ich behaupten, dass ich als Tomboy aufwuchs, damals kannte ich den Ausdruck noch nicht. Ich war stolz, wenn Unbekannte mich als Jungen identifizierten. Meine Eltern versuchten nicht, mich zu verbiegen. Mein Vater wünschte sich ja auch den Jungen, er unterstützte meine damals klassische männliche Ausbildung als Hochbautechnikerin und später zur Architektin. Er wollte unbedingt, dass ich den LKW-Führerschein mache, und noch später konnte ich ihm auch die Freu- 
de einer Ausbildung im Bereich der Forstwirtschaft bereiten. Und dennoch gab es da Grenzen. Menschen, die mir subtil oder weniger subtil klarmachten, dass ich da in eine fremde Domäne eindringe und hier nichts verloren habe. Diese Grenzen konnte ich mit 15 Jahren noch nicht strukturell erfassen. Aber durch die kontinuierliche Suche, unzählige Diskussionen, Bücher, viele besuchte Lehrveranstaltungen der in den 1990er Jahren aufkommenden Gender Studies ergibt sich für mich ein klareres Bild dessen, was den kleinen Unterschied des Geschlechts darstellt. Durch inhaltliche Fokusverschiebungen meiner Interessen von anfangs stark technischen Ausrichtungen, unter anderem auch Medienkunst, hin zu vermehrt geisteswissenschaftlichen, kunst- und kulturwissenschaftlichen Themenbereichen habe ich auch die Problemfelder zur Frage der Möglichkeiten emanzipatorischer und feministischer Lebenskonzepte kennengelernt, die, egal ob sie sich jetzt im Bereich der Naturwissenschaften oder der Geisteswissenschaften darstellen, höchst ähnliche sind, teilweise gänzlich austauschbare.

Uns allen ist gemein, dass wir feministisch unterrichten (wollen) und Gemeinsamkeiten beziehungsweise ähnliche Erfahrungen haben, die uns zu unserem interdisziplinären Austausch und zum Schreiben dieses Beitrags inspiriert und motiviert haben: Beim Einbringen feministischer Inhalte in die Regellehre respektive in Kunstprojekte sind und waren wir immer wieder mit Widerständen beziehungsweise negativen Reaktionen konfrontiert (Liebig 2009). Feministische Lehre findet in einem Kontext statt, in dem Frauenfeindlichkeit und Antifeminismus (wieder) salonfähig sind (Scambor und Jauk 2018). Es gibt Personen, die behaupten, dass es sich um ein überholtes Thema handelt und dass sie kein Interesse haben, sich damit zu beschäftigen. Auch Anne Schlüter und Nicole Justen (2009) sahen sich in ihrer Untersuchung damit konfrontiert, dass viele Studierende eine Abwehrhaltung gegenüber den Genderthemen haben. Durch gesellschaftliche Transformationsprozesse wie die Entöffentlichung und Dethematisierung geschlechtshierarchischer Widersprüche und die Individualisierung gesellschaftlicher Konflikte, die ihre Lösung zu einer Privataufgabe machen, werden geschlechtsbezogene Ungleichheit, Hierarchie und Benachteiligung verdeckt (vgl. Bitzan 2002, 30). Alle drei kennen wir das desinteressierte, manchmal rötlich-erregte Gesicht, wenn das F-Wort Feminismus - oft bewusst, manchmal unbewusst - in den akademischen Lern- und Forschungsraum gesetzt wird. Unsere subjektiven Erfahrungen sind eingebettet in gesellschaftliche Rahmenbedingungen und Geschlechterarrangements, die sich im Spannungsfeld von Wandel, Beharrung und (Re-)Traditionalisierung befinden (Riegraf 2015, 11f). Die Geschlechterverhältnisse sind in den letzten Jahren in widersprüchlicher Weise in Bewegung geraten: Zum einen scheint es zu einer allmählichen Aufweichung geschlechtsspezifischer Trennungslinien in der Berufs- und Arbeitswelt zu kommen (Kutzner 1999). Zum anderen ist eine erstaunlich stabile Persistenz und sogar Verfestigung zu beobachten (Wetterer 2002). 


\section{Gesellschaftsanalytische Rahmung unserer subjektiven Erfahrungen: Rhetorische Modernisierungsprozesse und ein neuer Geschlechtervertrag}

Die von uns (nicht nur in der Lehre) wahrgenommenen Zurschaustellungen des Nichtmehr-nötig-Habens beziehungsweise die Annahme des $\mathrm{Zu}$-weit-Gehens feministischer Politik (Fegter 2012) sind keine partikularen und individuellen Phänomene, sondern Teil aktueller gesellschaftlicher Entwicklungen, der auch theoretisch gefasst wurde und dem wir uns nun zuwenden. Als Referenzrahmen beziehen wir uns auf Überlegungen zu rhetorischen Modernisierungsprozessen (Wetterer 2003) und nehmen Bezug auf einen neuen neoliberalen Geschlechtervertrag (McRobbie 2010). Angelika Wetterer merkt hinsichtlich der Fragen nach der Modernisierung und der Relevanz des Geschlechterverhältnisses in der heutigen westlichen Gesellschaft an, dass die gegenwärtige Situation vor allem durch Widersprüche, Brüche und Ungleichzeitigkeiten gekennzeichnet sei (vgl. 2003, 288). Sie beschreibt ein Nebeneinander von Gleichheit und Ungleichheit und die Diskrepanz zwischen den Überzeugungen und dem Handeln der Individuen. Angelika Wetterer beschreibt mit dem Begriff der »rhetorischen Modernisierung « eine Neuerung, »die sich im Diskurs und der Sprache, kaum jedoch in der Praxis zeigt « $(2006,12)$. Dieser Widerspruch wird von den Individuen mit der Dethematisierung der Ungleichheit aufgelöst, welche dadurch jedoch nicht aus der Welt geschafft, sondern vielmehr vor Kritik geschützt wird. Die hierarchische Struktur der Geschlechterunterscheidung wird aus dem individuellen Erfahrungs- und Sprachrepertoire ausgeschlossen und damit unsichtbar gemacht (vgl. Wetterer 2003, 290). In Anlehnung an Cornelia Koppetsch und Günter Burkart (1999) nennt Angelika Wetterer die Mechanismen, auf die dabei zurückgegriffen wird, die »Logik der Diskurse « (Wetterer 2003, 298). Dabei wird die Ungleichheit als »Folge einer freien und bewusst getroffenen Wahl « (2003, 298) verstanden, für welche die Akteur*innen selbst verantwortlich seien; strukturell angelegte Probleme werden so personalisiert und individualisiert. Die paradoxe Situation besteht somit darin, dass während in der Diskurslogik eine Gleichheitsrhetorik vorherrscht, in der Praxis soziale Ungleichheiten fortbestehen können. Angelika Wetterer spricht in diesem Zusammenhang davon, dass Gleichberechtigung als »Regulativ des Redens « fungiert $(2013,247)$.

Um die Frage nach der theoretischen Rahmung unserer subjektiven Erfahrungen zu konkretisieren, haben wir auch die Überlegungen von Angela McRobbie herangezogen. Sie konstatiert in ihrer Studie Top Girls - Feminismus und der Aufstieg des neoliberalen Geschlechterregimes (2010), dass gegenwärtig jungen und gut ausgebildeten Frauen aus westlichen Ländern vonseiten neoliberaler Regierungsformen und Popkulturen ein »neuer Geschlechtervertrag « $(2010,57)$ und » eine Art rhetorische Gleichheit « $(2010,18)$ angeboten würden. Ihnen wird offeriert, an der Öffentlichkeit 
teilzuhaben, am Arbeitsmarkt erfolgreich zu partizipieren, sich weiterzubilden, selbstbestimmt einen oder auch keinen Kinderwunsch zu artikulieren und genug Geld zu verdienen, um an einer an Konsum orientierten Gesellschaft teilzuhaben (vgl. 2010, 37). Die damit (performativ) hervorgebrachten Frauen(-bilder) zeigen die erreichten Erfolge in der Gleichstellung der Geschlechter und legen nahe, dass feministische Interventionen und Kritik an (patriarchalen) Herrschaftsverhältnissen nicht mehr notwendig seien (vgl. Klinger 2014, 323). Im Gegenzug zur öffentlichen Sichtbarkeit wird von Frauen allerdings (implizit) erwartet, auf feministische Politik und Positionen zu verzichten (vgl. McRobbie 2010, 37). Zwar werden in dieser Vorgehensweise die Erfolge des Feminismus anerkannt - dies ist auch ein zentraler Unterschied zu traditionellen Backlash-Debatten -, doch feministische Gesellschaftskritik wird als unzeitgemäß abgetan. Den Verzicht auf feministische Inhalte und Forderungen nennt Angela McRobbie in Anlehnung an Stuart Hall »Politik der Desartikulation « (2010, 47ff.). Diese Überlegungen verbinden sich mit den von Susanne Maurer »Verheißungen des Neoliberalismus « $(2006,241)$ genannten Strukturen. Sie thematisieren das Aufgreifen und die Funktionalisierung emanzipatorischer, feministischer Anliegen, die diese auf die Frage nach Erwerbstätigkeit und Wettbewerbsfähigkeit reduzieren: So sind die hohen Erfolgsquoten bei der Erlangung von Qualifikationen zum Maßstab der bisher erreichten Gleichberechtigung geworden (vgl. McRobbie 2010, 113). Auch empirisch lässt sich nachzeichnen, dass bei der studentischen Auseinandersetzung mit Geschlecht und Geschlechterfragen auf den von Angela McRobbie konstatierten neuen Geschlechtervertrag (2010) sowie die »rhetorische Modernisierung « (Wetterer 2003) Bezug genommen wird und diese als Orientierungsrahmen rekonstruiert werden kann (Klinger 2014, 2015). Beide Konzepte scheinen den studiumsbezogenen und wissenschaftlichen Erfahrungsraum, in dem die akademische Sozialisation erfolgt, zu überlagern und zu dominieren.

\section{Autoethnografische Dialoge/Trialoge: Vom Suchen und Finden feministischer Lehrhaltungen und -praxen}

Wir gehen im Folgenden in einem dialogisch-autoethnografischen Prozess der Frage nach, was für uns Lehre ausmacht, die im Spannungsfeld von wissenschaftlichem Arbeiten und gesellschaftskritischem Impetus angesiedelt ist. Gleichzeitig gehen wir der Frage nach, ob und wie es beim Einbringen feministischer Inhalte in die Lehre und das wissenschaftliche Arbeiten Sinn machen kann, das F-Wort (Feminismus) gerade (nicht) offensiv zu verwenden und dennoch subversiv feministisch zu bilden und zu inspirieren. Unsere autoethnografische Narration ist nicht als fertige Analyse oder Forschungsergebnis zu verstehen und endet auch nicht mit der Produktion beziehungsweise Abgabe 
dieses Textes, sondern erst mit dem sinnlichen, emotionalen Erleben der jeweiligen Leser*innen (Ellis und Bochner 2000). Der Text ist somit als Verstehensprozess aufseiten der Schreibenden zu versehen, der auf Basis einer reflexiven Auseinandersetzung mit der Subjektivität der Autor*innen (Ploder und Stadlbauer 2013, 391) die Leser*innen dazu auffordert, durch die dargelegte Wirklichkeit und Wahrnehmung die erörterten Ergebnisse zu transformieren und mit zu produzieren (Ploder und Stadlbauer 2013, 378). Dahinter steht das Ziel feministischer Lehre, dass es neben der Vermittlung grundlegender Inhalte auch immer darum geht (Reflexions-)Räume und Möglichkeiten zu bieten, um über Hierarchien nachzudenken und diese zu brechen, und damit als Lehrende auch immer Lernende zu bleiben (hooks 2010). Für das Umsetzen und Ausdifferenzieren dieser Ziele respektive feministischer Lehrhaltungen und -praxen gibt es kein Rezeptwissen und auch keine einfachen Wenn-dann-Kausalitäten, sondern es handelt sich dabei um einen komplexen Prozess, der sich im permanenten Diskurs und Austausch mit anderen sowie in der omnipräsenten und -relevanten (Selbst-)Reflexion entwickelt. Im Folgenden werden wir anhand autoethnografischer Auszüge Einblicke in unseren gemeinsamen Prozess des Suchens und Findens feministischer Lehrhaltungen und -praxen geben. Hierfür diskutieren wir gleichsam dreistimmig die Frage, was feministische Lehre ausmacht, die im Spannungsfeld von wissenschaftlichem Arbeiten und gesellschaftskritischem Impetus angesiedelt ist.

Autor*in L: Für mich stellt sich immer wieder die Frage nach der Formation der Subjekte durch gesellschaftliche Diskurse. Somit eröffnet sich eine Perspektive auf das Zusammenwirken unterschiedlicher gesellschaftlicher Diskurse, mittels derer zentrale gesellschaftliche Normen transportiert werden und an denen sich die Individuen in ihren Lebens- und Existenzweisen orientieren (müssen). Laut Andrea Maihofer (2002) wird folgende wichtige subjektorientierte Frage entweder gar nicht mehr oder nur mehr in sehr reduzierter Form gestellt: Wie wird in »diesen konkreten Gesellschaftsverhältnissen unter Bedingungen eines hegemonialen Diskurses qualitativer, heterosexueller Geschlechterdifferenz aus einem kleinen Wesen eine erwachsene $>$ Frau $<$ (und) oder ein erwachsener $>$ Mann $<[\ldots]$ und wie $[\ldots]$ modifiziert $[$ sich] dies im Laufe des Lebens $\ll(2002,16)$ ? Diese Frage nach dem »Subjekt und seiner Geschichte« (Dausien 2006, 17), also nach dem »Gewordensein « von Geschlecht, hat die Art und Weise im Blick, wie in dieser Gesellschaft Individuen - die sich als Frau oder Mann » geworden « und »seiend « empfinden - existieren, sich verändern, handeln und denken (Maihofer 1995). Deshalb ist es für mich neben der Vermittlung theoretischer und fachlicher Inhalte auch wichtig, gemeinsam mit den Studierenden die Analyse der gesellschaftlichen Diskurse und deren Einfluss auf Wahrnehmungs-, Handlungs- und Bewertungsprozesse stärker zu berücksichtigen. Dies bedeutet, die gegenwärtigen gesellschaftlichen Diskurse und gesellschaftlichen Transformationsprozesse stärker in der theoretischen 
Konzeption wie auch der empirischen Forschung aufzugreifen. Dies bedenkend, kann in Zeiten neoliberaler Vereinnahmungen und Verkürzungen auch die Frage nach dem sozialisatorischen Horizont der (De-)Thematisierung von Geschlecht und damit zusammenhängend auch die Bedeutung von Feminismus und Feminismen (mit den Studierenden gemeinsam) neu verhandelt werden. In meiner Lehre versuche ich dies auf unterschiedlichen Ebenen umzusetzen. Hier steht die Vermittlung von theoretischem Fachwissen (a) ebenso im Zentrum wie die Berücksichtigung kritischer Gesellschaftsdiagnosen und geschlechterreflektierender und diversitätsbewusster Fachinhalte. Die theoretischen Bezüge variiere ich hier je nach Lehrveranstaltungsschwerpunkt. Dabei ist eine interaktive anstatt einer instruktiven beziehungsweise unidirektionalen Bearbeitung der Inhalte von großer Bedeutung. Zur didaktischen Vermittlung erscheinen mir unter anderen folgende Lehrmethoden besonders geeignet: Murmelgruppen, Rotierende Partner*innengespräche, Fishbowl-Diskussion, World-Café (The World Café Community Foundation 2018), Gender-Activity und Wissens-Staffellauf (Portmann 2015). Um feministische Inhalte vermitteln zu können, ist ein praxisorientierter $\mathrm{Zu}$ gang (b) für mich sehr wichtig. Als besonders bereichernd erleben Studierende die zu meinen Lehrveranstaltungen regelmäßig eingeladenen Gastvortragenden aus unterschiedlichen feministischen Kontexten und Kunstprojekten. Neben Gastvorträgen nutzen wir als didaktische Methoden fallbezogene kollegiale Beratung (Schlee 2012), soziometrische Aufstellungen (Ameln und Kramer 2014) und Beobachtungsaufträge, Analyse von Zeitungsartikeln und Youtube-Videos. Zudem habe ich mit der Integration einer biografieorientierten Herangehensweise in der Lehre besonders gute Erfahrungen gemacht. Insbesondere wenn es um den Umgang mit Widerständen geht, ist diese selbstreflexive Ebene und die wertfreie Befragung der jeweiligen Lebensrealitäten ein wichtiges Hilfsmittel. Das Bewusstmachen von Denk- und Handlungsmustern sowie das Heben und Nutzbarmachen lebensgeschichtlicher Ressourcen sind dabei wichtig, da soziale Fragen sowie Aspekte der Erziehung und Bildung biografischen Einflüssen unterliegen, die häufig unbewusst bleiben. Es steht dabei aber nicht nur das Individuum selbst im Zentrum, sondern auch gesellschaftliche, historische und kulturelle Kontexte werden lernwirksam. Um dies zu ermöglichen, arbeiten wir in den Lehrveranstaltungen je nach Schwerpunkt mit unterschiedlichen didaktischen Lehrarrangements, zum Beispiel Kartenspiel-Barnga (Local Players o.J.), Privilegientest (Portal Intersektionalität; Bergische Universität Wuppertal o. D.), angeleitete Selbstreflexion (Mafalda 2014), Freewriting (Wolfsberger 2010), Biografische Interviews (Kirchhof 2008), Autoethnografie (Ploder und Stadlbauer 2013).

Autor ${ }^{*}$ in O: Meine Handlungen und mein Denken sind wesentlich geprägt von der Biologin Anne Fausto-Sterling. Anne Fausto-Sterling hat meine Sichtweise auf Geschlechtsidentität maßgeblich geformt. Ihre Konzeptionierung eines multidimensiona- 
len Geschlechtsraumes Anfang der 1990er Jahre kennt keine Polbildung wie männlich oder weiblich, Sex und Gender, sondern hat ein vieldimensionales Achsensystem (z. B. genetisch, zellulär, hormonell, anatomisch, Umwelteinflüsse, Erfahrungen, Alter etc.) (Fausto-Sterling 1993, 2000). Die Auflösung der Kategorisierungen, aber auch das verqueeren unserer Identitäten bieten enorm wichtige Anhaltspunkte, um allen unterschiedlichen Geschlechtern ihre Menschlichkeit zu gewährleisten und die Möglichkeit geben zu können, ihre friedvollen Auseinandersetzungen miteinander auszuleben. Ich arbeite im Moment an einem umfangreicheren interdisziplinären Kunst-, Forschungsund Friedensprojekt, welches sich mit Demokratie und Frieden auf der Straße auseinandersetzt (vgl. https://comradeconrade.mur.at). Mein Startpunkt war, eine wirklichkeitsgerechtere Sichtbarkeit von unterschiedlichen Geschlechtern im öffentlichen Raum zu thematisieren und zu forcieren. Hier geht es um Würde, ein Leben ohne Herabwürdigung und vor allem auch darum, Grenzüberschreitungen anzusprechen. Es geht darum, alle Menschen als vollwertige Menschen zu sehen und zu hören, intersektional mit unseren jeweiligen Geschlechtsidentitäten und Herausforderungen. Mit dem, wie wir sind und was wir können, sind wir in der Lage, gemeinsam eine positive $\mathrm{Zu}$ kunftsvision zu entwickeln, aber dazu müssen alle Geschlechter dialogisch hereingeholt werden. In der Arbeit mit Studierenden, aber nicht nur dort, versuche ich diskursive Räume aufzumachen, die nicht in starren Gegenpositionen enden, sondern im besten Fall eine konstruktive Resonanz (Rosa 2016) hervorrufen. Projektorientiertes und forschungsgeleitetes Arbeiten bietet hier gute Möglichkeiten. Im Kontext der Lehre ist es für mich wichtig, einen sicheren Raum aufzumachen und diesen dann auch durchgängig geschützt und reflexiv zu halten. Verletzungen und gewaltvolle Erfahrungen (zu denen ich auch sprachliche Unterdrückungsprozesse zähle), die innerhalb von geschützten Räumen passieren, sind noch viel erniedrigender als anderswo, da sie innerhalb der scheinbaren Sicherheit erfolgen. Nicht nur innerhalb meiner Lehre bin ich überzeugt davon, mit konstruktiven Kritiken und einem »Im-Gespräch-Bleiben « herausfordernde (gesellschaftliche) Konfliktsituationen lösen zu können, die nicht immer, aber oft auf fehlender Geschlechtergerechtigkeit oder Dethematisierung von unterschiedlichen Bedürfnissen unterschiedlicher Geschlechter fußen.

Autorin X: Ich bin inspiriert von Halberstams Gagafeminismus (für eine Zehn-Minuten-Videoperformance zu gagafeminist didactics siehe Jauk et al. 2017). Die »Kunst des Gaga « ist »eine Politik des freien Falls, wilden Denkens, und der fantasievollen Neuerfindung, die am besten von Kindern unter Acht, Frauen über 45 und den Armeen marginalisierter, verlassener und unproduktiver Menschen repräsentiert wird « (Halberstam 2012, xv). Das heißt für uns, die wir Lernen möglich machen sollen, selbst an nichtnormativen Orten zu lernen, aber Studierenden auch zu erlauben, die Ränder und Ungewöhnliches zu untersuchen, um die soziale Welt rund um sie besser 
zu verstehen. Also: Was wäre, wenn wir hinausgehen und an einem sozialen Protest teilnehmen, anstatt die 67. PowerPoint-Folie zu projizieren? Lasst uns auch ungewöhnliche Lernorte in unsere Klassenräume bringen. Das heißt, Verbindungen herzustellen zu sozialer Praxis und die soziale Welt in unsere Lernumgebungen zu bringen durch Gastreferent*innen und Aktivist*innen, kurz: auch Platz zu machen für Menschen, die man typischerweise nicht an der Universität sieht und auch ihnen unsere Kursräume zugänglich zu machen. Lernen für uns heißt auch, Lehrbücher um mehr Lernmaterialien zu erweitern, zum Beispiel um Kunst, Artefakte, Multimedia. Und das heißt auch, technik- und communitypositive Feminismen auf vielen Wegen in das Klassenzimmer zu holen: Das können Gastreferent*innen sein, die aus Guatemala eingeskypt werden, das können Cyberfeministinnen sein, die uns online feminist hacking beibringen, das kann feministische Netzkunst sein, das sind lokale Farmer*innen, die food insecurity bekämpfen und biologische Tomaten als Snack bringen, das sind queere Aktivist*innen, die ein von ihnen entwickeltes Theaterstück im Unterrichtsraum aufführen. Als feministische Lehrende möchte ich auch Studierende in die Communitys bringen (auf ressourcensensitive Weise, sodass sie beitragen, nicht wegnehmen oder belasten). Ich möchte Lernräume ko-kreieren und den Studierenden als Lerner*in begegnen, die sich bewusst ist, dass sie Verantwortung trägt für gerechten Informationsinput, aber kein mechanischer und konsumorientierter »Lektionsautomaton « ist. Ich möchte Fragen evozieren und aushalten, dass Feminismen nicht im Entweder-oder, sondern im Sowohl-als-auch existieren. Ich möchte mich und andere(s) hinterfragen dürfen.

In den gemeinsamen Gesprächen, Dialogen und Reflexionen der Autor*innen stand auch die Umsetzung unserer feministischen Lehrhaltung im Zentrum. Dabei teilten wir die Erfahrungen, immer wieder mit Widerständen, Abwehrhaltungen beziehungsweise negativen Reaktionen konfrontiert zu sein. Unterschiedlich war hingegen unser individueller und kontextabhängiger Umgang mit dem F-Wort, welcher in einem regen und interessierten Austausch mündete. Die Essenz dieses Diskurses wird entlang folgender Frage dargestellt: Wie kann eine feministische Lehrhaltung und -praxis aussehen, die mit und/oder ohne den Begriff »Feminismus « respektive »Feminismen « auskommt? Wie sieht der Umgang mit dem F-Wort aus?

Autorin X: Ich bin in den meisten Lernräumen, in denen ich unterrichtet habe, sehr klar und stecke mir das F-Wort an den Hut. Ich trage es vor mir her, ich spreche es aus, ich setze es in die Mitte des Raumes am ersten Tag. Ich behandle es wie einen Diamanten, ich bin stolz auf Feminismen und all die denkenden Praktiker*innen, die Wege für uns aufbereitet haben. Ich finde es wichtig, transparent und authentisch zu sein, und »feministisch « ist, was ich BIN, was ich immer war, auch wenn sich Bedeutungen des Wortes verändert haben und immer multipel sind. Ich mache mich angreifbar und ich 
mache Werbung. Wenn Studierende das » rötlich-erregte Gesicht« bekommen, dann bekomme ich manchmal Angst, aber ich habe mich noch nie geschämt. Dann ist es so wunderbar und hilfreich, alle daran zu erinnern, dass wir in der Wissenschaft verankert sind und evidenzbasiert arbeiten. Her mit den Daten! Her mit der systematischen Forschung. Meine Teamteacherkolleg*in Dr. Solveig Haring hat mich im gemeinsamen Lehren gelehrt, in der ersten Einheit klarzumachen, dass »Privatstatistiken « in diesem Lernraum nicht willkommen sind. Das sind die einzelnen Beispiele und Ausnahmen, die wir alle kennen. Etwa die Nachbarin, die mehr verdient als ihr Ehemann, der seinerseits jede Woche das Haus reinigt. Oder der Opa, der 50 Jahre geraucht hat und keinen Lungenkrebs bekam. Als Wissenschaftler*in will ich Daten sehen. Ich will Muster erkennen helfen. Und dabei auch eines klarmachen: Lohnunterschiede, Gewalt, Geschlechterstereotypen, das binäre Geschlechtersystem als Zwangskorsett für alle Menschen - da geht es nicht nur um Frauen. Feminismus ist für alle gut. Aber ich hatte auch das Privileg, in Soziologie und Geschlechterforschung zu unterrichten, unter der Käseglocke. Ich bin mir sicher, in Architektur und Technik trifft man auf viele rötlich-erregte Gesichter und untergräbt vielleicht eigene Autorität mit dieser offensiven F-Wort-Strategie. Wenn ich im Lernraum offensiv feministisch »performiere « und Machtunterschiede in den Mittelpunkt aller Themen stelle, dann nehme ich den Studierenden vielleicht auch das »Aha-Erlebnis « des Entdeckens, die Überraschung, die da ist, wenn mensch Daten verstehen lernt, und sieht, dass »Gender « manchmal buchstäblich Leben und Lebenschancen zerstört und Leben verhindert (Stichwort: selektive Abtreibungen, Femizid).

Autor*in L: In meiner Lehre gehe ich mit meiner eigenen feministischen Grundhaltung eher defensiv um. Auch das F-Wort wird von mir vor allem zu Beginn der Lehrveranstaltungen selten verwendet. Aus meiner Erfahrung ist Feminismus - Feminismen sind kaum bekannt - für Studierende etwas Verstaubtes, das obsolet geworden ist, obendrein noch nervt und nur vereinzelt auf Zuspruch stößt. Um die Studierenden also sozusagen nicht mit dem gleisenden und hellen Licht des feministischen Diamanten in der Mitte des Raumes (siehe oben) zu blenden, favorisiere ich es, vor allem feministische Prinzipien und Anliegen (z. B. Solidarität, Hinterfragen von Normativitäten, Aufzeigen struktureller Ungleichheit, die Lebenswelten der Studierenden ernstnehmen, Intersubjektivität, Emanzipation) zu vermitteln und nach diesen zu agieren. Dabei stehen unterschiedliche Kategorien sozialer Ungleichheit (Klasse, Rasse, Gender, Alter etc.) im Zentrum. Hier greife ich auch gerne auf didaktische Strategien der geschlechterreflektierten Pädagogik zurück. Dabei handelt es sich um die Dramatisierung, die Entdramatisierung und die Nichtdramatisierung von Geschlecht (Debus 2012, 150f.). Dramatisierende Herangehensweisen sind unter anderem sinnvoll, wenn die pädagogische Arbeit die Teilnehmenden zum Nachdenken über Geschlechterverhältnisse anregen soll. Durch eine entdramatisierende Herangehensweise kann sichtbar 
gemacht werden, dass Geschlecht nicht die einzige Differenzkategorie ist. Die Strategie der Nichtdramatisierung zeichnet sich dadurch aus, dass sie Geschlecht unter anderem als Analyseansatz im Hinterkopf behält, es aber nicht in den Mittelpunkt der pädagogischen Aktivitäten stellt. Nichtdramatisierende Angebote beginnen in einem Raum, in dem Geschlecht nicht als zentral gesetzt ist. Ziele dieser Strategie sind: Förderung individueller Vielfalt und individueller Kompetenzen, Auseinandersetzung mit anderen Themen als Geschlecht (Debus 2012, 155). Das kann auch bedeuten, immer wieder aufs Neue infrage zu stellen, ob bestehende Kategorien der Komplexität menschlicher Lebensrealitäten entsprechen, und die Frage nach » anderen « Kategorien zu stellen und somit diverse Relevanzsysteme mit einzubeziehen. Mir ist es wichtig, einen grundlegenden gesellschaftlichen Bezug herzustellen, der mit den gesamten Lebenserfahrungen der Studierenden verwoben ist. Zudem ist es mir wichtig, begeisternd zu bilden und nicht (immer) »kämpferisch und ernst « zu überzeugen. Mein Ziel ist es, die Normativität gesellschaftlicher Kompetenz- und Handlungserwartungen nicht ungebrochen zu transportieren. In meinen Lehrveranstaltungen gilt es, diese Normen selbst kritisch zu hinterfragen und diese gegebenenfalls in Bezug auf eine alternative Lebensführung und eine humanere Welt zu verändern. Spätestens wenn diese »subversive « Strategie Früchte trägt und die Studierenden ihre eigenen Vorstellungen und Vorurteile aufgrund der geführten inhaltlichen Diskussionen zumindest einmal infrage stellen wollen und können, ist es für mich Zeit, mir das F-Wort stolz, offensiv und mit Begeisterung an meinen Hut zu heften. Von den Studierenden verlange ich in diesem Zusammenhang, sich einen Abstand zur umgebenden Welt zu verschaffen und sich auf ein ReflektierendAbwägendes einzulassen sowie auf möglicherweise Neues und Unbekanntes.

Autorin ${ }^{*} O$ : Aus meiner Erfahrung ist es wichtig, feministischen Inhalten in der Lehre treu zu bleiben. Das heißt, die beständige Einforderung feministischer Thematiken ist notwendig, da sie sonst leicht zu verschwinden drohen, nicht aus Absicht, sondern auch aus Gewohnheit, da sie sonst eben oft auch nicht vorhanden sind. Beispielsweise habe ich viele Jahre mit definitiv nicht feministisch Agierenden zusammengearbeitet, auch in Lehrräumen. Mir war hier wichtiger, eine Bevorzugung eines Geschlechts - sei es jetzt auf Studierendenseite, sei es in der Auswahl der Anschauungsbeispiele - nicht zuzulassen und aktiv eine feministische Haltung zu zeigen. Dabei auch noch zu proklamieren, offen zu sagen, dass es aus einer feministischen Überzeugung heraus kommt, hätte mehr Probleme als Gewinne gebracht. Wobei dies nicht bedeutet, dass ich immer eine subversivere Gangart wähle. Das ist kontextabhängig. Ich habe die Erfahrung gemacht, dass ich in manchen Kontexten einfach kein Gehör mehr finde, wenn ich offensiv formuliere. Und mir ist es wichtig, in einen Dialog zu kommen, um mit einem Gegenüber Austausch haben zu können, nicht Konfrontation. Für mich macht es Sinn, subversiv $\mathrm{zu}$ arbeiten, da es manchmal schlicht unmöglich ist, es nicht zu tun! Gayatri Spivaks 
»Strategischer Essentialismus « (Spivak 1996) scheint hier eine mögliche Antwort, um handlungsfähig zu bleiben. Dabei geht es Spivak darum, dass es politisch notwendig sein kann, sich in Identitäten hineinzudenken, aus strategischer Perspektive, um diese Identitäten als falsch zu entlarven und auf den Konstruktionscharakter hinzuweisen. Das reflektierte Handeln ist hier oberste Prämisse.

\section{Abschließende Gedanken und mehr offene Fragen}

Im Sinne einer kollektiven Wissensgenerierung möchten wir zusammenfassend festhalten, dass es für uns auch immer wieder zu hinterfragen gilt, inwiefern wir feministische Diskurse legitimieren und normalisieren und sie damit zu Instrumenten der Macht und Ermächtigung machen, indem wir radikale Wissensstränge kanonisieren. Stattdessen versuchen wir, diese in selbstreflexiver Praxis zu denaturalisieren und bewusst Fragen offenzulassen, wie auch die Beiträge von Luke und Gore (2014) aufzeigen. Wenn es ein vorläufiges Resümee unserer Trialoge gibt, dann ist es nicht die eine Antwort auf die Frage, was genau feministische Lehre ist, oder die Forderung danach, dass es ausschließlich profeministische Lehre geben soll. Wichtiger erscheint es uns, dass es keine antifeministischen oder misogynen oder xenophoben Haltungen und Positionen in der Lehre und Wissenschaft geben sollte, die empirische Ergebnisse und im wahrsten Sinne des Wortes Wissenschaft aus der Ungleichheitsforschung ignorieren oder verzerrt darstellen. Dennoch besteht Einigkeit darüber, dass wir feministische Lehre brauchen, da wir uns in einem politischen Klima bewegen, das von Radikalisierung, legitimiertem Antifeminismus und sozialer Ungleichheit geprägt ist.

Wir sind in der Lehre mit Entsolidarisierung aufgrund der rhetorischen Sättigung von Gleichheit und Gender (Klinger 2014) konfrontiert und herausgefordert. Feminismus und Feminismen sollten dadurch verstärkt in der Lehre aufgegriffen werden, auch um Möglichkeiten der reflektierenden Auseinandersetzung auf mehreren Ebenen zu schaffen und somit mit Widerständen umzugehen zu lernen. Dazu ist es nötig, dass wir uns formell und informell auch weiterhin austauschen. Dieser Beitrag ist ein Versuch, beides simultan zu tun. Das systematische Erfahren und Analysieren unserer autoethnografischen Trialoge hat sich dabei als ideales Vehikel für unseren Austausch erwiesen, der ganz im Sinne der Methode zugleich Prozess und auch Produkt ist. Zudem hat die Autoethnografie auch didaktisches Potenzial, das von uns noch erprobt werden soll. Sie bietet den Studierenden die Gelegenheit, sich mit dem eigenen Erleben als Dokument von Gesellschaft auseinanderzusetzen und so den Einsatz des eigenen Ichs als Forschungsinstrument zu schulen und gleichzeitig die Macht- und Herrschaftsverhältnisse unter einem emanzipatorischen Anspruch kritisch zu reflektieren (Ploder und Stadlbauer 2013, 403). 
Da dieser Prozess niemals abgeschlossen ist, bleiben auch am Ende dieses Beitrags noch viele Fragen offen. Zum Beispiel die Frage nach der Ambivalenz von Lehrenden wie uns, die wir in einem System arbeiten, das wir auch gleichzeitig kritisieren und verändern wollen. Meyerson und Scully (1995) nennen solche Individuen »tempered radicals $\ll$. Es ist radikal, das System verändern zu wollen, aber mensch muss »temperiert « vorgehen, also oft strategisch und mit Maß. Im Englischen heißt »tempered « auch »gehärtet, gestählt «. Eine dritte Interpretation ist die von »temper « als »Wut.« Meyerson und Scully (1995) sehen dabei die Ambivalenz nicht als Problem, das »gelöst « werden muss, sondern als Ressource, die nuanciertes Vorgehen zulässt. Es ist möglich, ambivalent zu sein und gleichzeitig sehr klar über eigene Identitäten und Werthaltungen. In diesem Sinne möchten wir auch weiter reflektieren über unsere eigene Ambivalenz dem F-Wort gegenüber. Es taucht zum Beispiel die berechtigte Frage auf, ob es paternalistisch sei, den Lernenden das F-Wort vorzuenthalten, und welches Bild von Lehre als strategischer Interaktion denn einer subversiven Strategie zugrunde liegen könnte. Neben dem bereits erwähnten »Strategischen Essentialismus « (Spivak 1996) als subversive und strategische Perspektive könnte auch eine weiterentwickelte Form von Gagafeminismus einen Weg weisen, der Raum macht für ein kreatives Kombinieren von Methoden und Didaktiken. Denn Gagafeminismus ist » a scavenger feminism that borrows promiscuously, steals from everywhere, and inhabits the ground of stereotype and cliche all at the same time« (Halberstam 2012, 5). In einem gagafeministischen Rahmen können wir »spielen«, ausprobieren, Fehler machen und experimentieren (wie etwa in diesem Artikel), denn » $[\mathrm{g}]$ aga feminism [...] is a form of political expression that masquerades as naive nonsense but actually participates in big and meaningful forms of critique « (Halberstam 2012, xxv). Offen bleiben viele Fragen. - Und das ist gut so. - Das ist gewollt so - unsere Trialoge gehen weiter.

\section{Literatur}

Ameln, Falko und Josef Kramer. 2014. Psychodrama: Grundlagen. 3. Aufl. Berlin \& Heidelberg: Springer Verlag.

Bergische Universität Wuppertal. o. D. »Portal Intersektionalität«. Zugegriffen am 17.02.2014. http:// portal-intersektionalitaet.de/startseite.

Bitzan, Maria. 2002. „Sozialpolitische Ver- und Entdeckungen. Geschlechterkonflikte und Soziale Arbeit«. WIDERSPRÜCHE. Zeitschrift für sozialistische Politik im Bildungs-, Gesundheits- und Sozialbereich 84 (22): 27-42.

Dausien, Bettina. 2006. »Geschlechterverhältnisse und ihre Subjekte. Zum Diskurs um Sozialisation und Geschlecht«. In Sozialisation und Geschlecht. Theoretische und methodologische Perspektiven, hrsg. v. H. Bilden und B. Dausien, 17-44. Opladen \& Flamington Hills: Verlag Barbara Budrich.

Debus, Katharina. 2012 »Dramatisierung, Entdramatisierung und Nicht-Dramatisierung in der Geschlechterreflektierten Bildung. Oder (Wie) Kann ich geschlechterreflektiert arbeiten, ohne 
geschlechtsbezogene Stereotype zu verstärken?«. In Geschlechterreflektierte Arbeit mit Jungen an der Schule, hrsg. v. Dissens e. V., Katharina Debus, Bernhard Könnecke, Klaus Schwerma und Olaf Stuve, 150-58. Berlin: Dissens e. V. Zugegriffen am 07.09.2015. http://www.dissens. de/de/publikationen/jus.php.

Crabtree, Robbin und David Allen Sapp. 2003. »Theoretical, political, and pedagogical challenges in the feminist classroom: Our struggles to walk the walk«. College Teaching 51 (4): 131-40.

Ellis, Carolyn und Arthur Bochner. 2000. »Autoethnography, Personal Narrative, Reflexivity. Researcher as Subject «. In Handbook of Qualitative Research, hrsg. v. N. K. Denzin und Y. Lincoln, 733-68. London: Sage.

Ellis, Carolyn, Tony E. Adams und Arthur P. Bochner. 2011. »Autoethnography: an overview«. Historical Social Research/Historische Sozialforschung 12 (4): 273-90.

Fausto-Sterling, Anne. 1993. »The Five Sexes«. The Sciences (März/April): 20-25.

Fausto-Sterling, Anne. 2000. »The Five Sexes, Revisited«. The Sciences (Juli/August): 18-23 [frz. Übers. 2001-2002. »La fin programmée du dimorphisme sexuel«. La Recherche 6: 58-62].

Fegter, Susanne. 2012. »Oder müsste ihnen nur mal jemand richtig zuhören?« Eltern, Schule und Gesellschaft als Adressaten im Mediendiskurs um Jungen als (Bildungs-)Verlierer«. In Jungen - Pädagogik. Praxis und Theorie von Genderpädagogik, hrsg. v. M. Diaz, S. Fegter, U. Graff und T. Doro, 34-48. Chwalek \& Wiesbaden: Springer VS.

Halberstam, Jack. 2012. Gaga feminism: Sex, gender, and the end of normal. Boston: Beacon Press.

Haraway, Donna. 1988. „Situated knowledges: The science question in feminism and the privilege of partial perspective«. Feminist studies 14 (3): 575-99.

Hesse-Bibber, Sharlene N. und Deborah Piatelli. 2012. »The feminist practice of holistic reflexivity«. In Handbook of feminist research: Theory and praxis, hrsg. v. Sharlene N. Hesse-Biber, 557-582. Thousand Oaks, CA: SAGE Publications. https://doi.org/10.4135/9781483384740.n27.

hooks, bell. 1994. Teaching to Transgress: Education as the Practice of Freedom. New York \& London: Routledge.

hooks, bell. 2010. Teaching Critical Thinking: Critical Wisdom. New York \& London: Routledge.

Jauk, Daniela und Nicole Pruckermayr. 2016. "Sex (and Genders) in der Seestadt - (De!-)Konstruktionen von Geschlecht im öffentlichen urbanen Raum«. fiber. werkstoff für feminismus und popkultur 25: 117-19.

Jauk, Daniela, Sabine Klinger und Nicole Pruckermayr. 2016. »Working with/out the F-Word: Interdisziplinäre Konzeptionen und Interventionen in der feministischen Lehre». Interaktiver Vortrag bei der Österreichischen Gesellschaft für Geschlechterforschung (ÖGGF) - Austrian Society for Gender Research, Österreich.

Jauk, Daniela, Solveig Haring und Anita Mörth. 2017 mbloody mary hairy! a DIY (gaga-)feminist didactics cock*tail tale«. Videoperformance zu »Gagafeminist Teaching«. Zugegriffen am 05.06.2018. https://www.youtube.com/watch?v=ThWI01zO-Fc\&t=353s.

Kirchhof, Steffen. 2008. Biografisch lernen \& lehren. Möglichkeiten und Grenzen zur Entwicklung biografischer Kompetenz. Flensburg: University Press.

Klinger, Sabine. 2014. (De-)Thematisierung von Geschlecht. Rekonstruktionen bei Studierenden der Erziehungs- und Bildungswissenschaften. Opladen, Berlin \& Toronto: Barbara Budrich.

Klinger, Sabine. 2015. »Die (De-)Thematisierung von Geschlechterhierarchien im Verhältnis akademischer Sozialisationsprozesse und gesellschaftlicher Diskurse«. In Geschlecht - Sozialisation - Transformationen. Jahrbuch der Frauen- und Geschlechterforschung in der Erziehungswissenschaft, hrsg. v. B. Dausien, C. Thon und K. Walgenbach, 111-28. Opladen, Berlin \& Toronto: Barbara Budrich.

Koppetsch, Cornelia und Günter Burkart. 1999. Die Illusion der Emanzipation. Zur Wirksamkeit latenter Geschlechtsnormen im Milieuvergleich. Konstanz: UVK Verlag. 
Kutzner, Edelgard. 1999. Die Un-Ordnung der Geschlechter. Industrielle Produktion, Gruppenarbeit und Geschlechterpolitik in partizipativen Arbeitsformen. München: Rainer Hampp Verlag.

Liebig, Brigitte. 2009. Gender Studies als Ausbildungsgang: Berufsqualifikation und Orientierungswissen. In Gender Studies in Ausbildung und Arbeitswelt. Das Beispiel Schweiz., hrsg. v. B. Liebig, M. Dupuis,T. Ballermer-Cao, A. Maihofer, 77-92. Zürich: Seismo Verlag.

Local Players. o.J. »Handbuch. Zur Nachahmung gedacht für Profis gemacht«. Zugegriffen am 18.09.2019. https://www.vielfalt-mediathek.de/data/handbuch_local_players.pdf.

Luke, Carmen und Jennifer Gore. 2014. Feminisms and critical pedagogy. New York, NY: Routledge.

Mafalda. 2014. »Meine Erfahrungen, Interessen und Stärken. Methoden \& Materialien zur Arbeit mit Kompetenzen«. Zugegriffen am 07.03.2015. https://www.mafalda.at/dafje746ne/ wp-content/uploads/meine_erfahrungen.pdf.

Maher, Frances und Mary Kay Thompson Tetreault. 2001. The feminist classroom: Dynamics of gender, race, and privilege. Lanham, MD: Rowman \& Littlefield.

Maihofer, Andrea. 1995. Geschlecht als Existenzweise. Macht, Moral, Recht und Geschlechterdifferenz. Frankfurt a. M.: Ulrike Helmer.

Maihofer, Andrea. 2002. »Geschlecht und Sozialisation. Eine Problemskizze«. Erwägen Wissen Ethik 13 (1): 13-26.

Maurer, Susanne. 2006. „Gouvernementalität ıvon unten her denken. Soziale Arbeit und soziale Bewegungen als (kollektive) Akteure sbeweglicher Ordnungen«. In Gouvernementalität und Erziehungswissenschaft: Wissen - Macht - Transformation, hrsg. v. S. Maurer und S. Weber, 233-52. Wiesbaden: VS Verlag für Sozialwissenschaften.

Messerschmidt, Astrid. 2011. »Lehrer/in sein in der Einwanderungsgesellschaft - Pädagogische Reflexion der gesellschaftlichen Bedingungen für den Umgang mit Migration«. $k p b$ 76: 105-14.

Meyerson, Debra und Maureen Scully. 1995. »Tempered Radicalism and the Politics of Ambivalence and Change«. Organization Science 6 (5): 585-600.

McRobbie, Angela. 2010. Top Girls. Feminismus und der Aufstieg des neoliberalen Geschlechterregimes. Wiesbaden: VS Verlag.

Ortner, Rosemarie und Elisabeth Hammer. 2015. »Involviert-Sein in gesellschaftliche Verhältnisse«. In facing differences. Materialien für differenzsensible Vermittlung in pädagogischer Aus- und Weiterbildung, hrsg. v. A. Czejkowska, R. Ortner und M. TUswald, 26-46. Wien: Löcker.

Ploder, Andrea und Johanna Stadlbauer. 2013. »Autoethnographie und Volkskunde? Zur Relevanz wissenschaftlicher Selbsterzählungen für die volkskundlich-kulturanthropologische Forschungspraxis«. Österreichische Zeitschrift für Volkskunde 116 (3/4): 373-404.

Portmann, Rosemarie. 2015. Die 50 besten Spiele für mehr Genderkompetenz. München: Don Bosco.

Radio Helsinki. 2017. „Working with/out the F-Word: Interdisziplinäre Konzeptionen und Interventionen in der Lehre«. Interview der Autor*innen mit Reni Hofmüller, Hotel Passage. Produziert am 24.01.2017, veröffentlicht am 15.02.2017. https://cba.fro.at/334546.

Riegraf, Birgit. 2015. »Konstruktionen der Geschlechterdifferenz in Bewegung: Wandel, Beharrung und (Re-)Traditionalisierung?«. In Des eigenen Glückes Schmied_in!?, hrsg. v. C. Micus-Loos und M. Plößer, 11-26. Wiesbaden: Springer.

Rosa, Hartmut. 2016. Resonanz: Eine Soziologie der Weltbeziehung. 2. Aufl. Berlin: Suhrkamp.

Scambor, Elli und Daniela Jauk. 2018. »Mander es isch Zeit - Akteur_innen, Denkformen, Diskurse und Argumente antifeministischer Männerarbeit in Österreich«. In Antifeminismus in Bewegung. Aktuelle Debatten um Geschlecht und sexuelle Vielfalt, hrsg. v. U. Peter, J. Lang und R. Claus. Hamburg: Marta Verlag.

Schlee, Jörg. 2012. Kollegiale Beratung und Supervision für pädagogische Berufe. Hilfe zur Selbsthilfe. Ein Arbeitsbuch. Stuttgart: Kohlhammer. 
Schlüter, Anne und Nicole Justen. 2009. »Pädagogische Biographiearbeit mit Studierenden zur Förderung der Genderkompetenz". In Gender als Indikator für gute Lehre. Erkenntnisse, Konzepte und Ideen für die Hochschule, hrsg. v. N. Auferkorte-Michälis, I. Stahr, A. Schönborn und I. Fitzek, 169-79. Opladen \& Farmington Hills: Budrich UniPress.

Sprague, Joey. 2005. Feminist Methodologies for Critical Researchers. Lanham: Rowman \& Littlefield.

Spivak, Gayatri. 1996. The Spivak Reader: Selected Works of Gayatri Chakravorty Spivak. London \& New York: Routledge.

Wetterer, Angelika. 2002. Arbeitsteilung und Geschlechterkonstruktion. „Gender at Work in theoretischer und historischer Perspektive. Konstanz: UVK-Verlag.

Wetterer, Angelika. 2003. »Rhetorische Modernisierung: Das Verschwinden der Ungleichheit aus dem zeitgenössischen Differenzwissen«. In Achsen der Differenz. Gesellschaftstheorie und feministische Kritik II, hrsg. v. G. Knapp und A. Wetterer, 286-319. Münster: Westfälisches Dampfboot.

Wetterer, Angelika. 2006. Ordentlich in Unordnung? Widersprüche im sozialen Wandel der Geschlechterverhältnisse. Österreichische Zeitschrift für Soziologie. 31. 5-22.

Wetterer, Angelika. 2013. »Das erfolgreiche Scheitern feministischer Kritik. Rhetorische Modernisierung, symbolische Gewalt und die Reproduktion männlicher Herrschaft«. In Gesellschaft. Feministische Krisendiagnosen, hrsg. v. E. Appelt, B. Aulenbacher und A. Wetterer. Münster: Westfälisches Dampfboot.

Wolfsberger, Judith. 2010. Frei geschrieben:Mut, Freiheit und Strategie für wissenschaftliche Abschlussarbeiten. Köln: UTB.

The World Café Community Foundation. 2018. »The WorldCafé«. Zugegriffen am 03.09.2018. http:// www.theworldcafe.com.

\section{Die Autorinnen}

Sabine Klinger, Mag.a phil., Dr.in phil., M. A., ist Universitätsassistentin am Institut für Erziehungsund Bildungswissenschaft der Universität Graz. Ihre Arbeitsschwerpunkte liegen in den Bereichen Diversität und Intersektionalität, geschlechterreflektierende Sozialpädagogik, Digitalisierung und Soziale Arbeit, Geschlechterforschung, und interpretativ-rekonstruktive Sozialforschung.

Kontakt: sabine.klinger@uni-graz.at

Daniela Jauk, Ph.D. ist assistant professor am Department for Sociology and Department of Criminal Justice and der University of Akron, Ohio. Sie forscht und lehrt in den Bereichen Geschlechterforschung, Kriminalsoziologie, und qualitative Sozialforschungsmethoden.

Kontakt:djauk@uakron.edu

Nicole Pruckermayr, Dlin Dr.in, ist freischaffende Künstlerin, Theoretikerin, Kuratorin, Forstarbeiterin und Mutter. Bevorzugt arbeitet und denkt sie mit sozialen sowie physischen Räumen/Orten, den handelnden Menschen/menschlichen Körpern, ihren Geschlechtern und Bedürfnissen. Ein besonderes Interesse gilt Handlungsfähigkeiten auf verschiedensten Ebenen.

Kontakt: nap@umlaeute.mur.at 